\title{
The Use of Terminalia arjuna as a Tonic
}

\section{Shukla Dhruti}

Department of Botany, Khyati Institute of Science, Ahmedabad, Gujarat, India

Corresponding author: dhrutianand@gmail.com

Received: 17-09-2019

Revised: 24-10-2020

Accepted: 29-11-2020

\begin{abstract}
Terminalia arjuna (Roxb. ex DC.) Wight \& Arn. (T. arjuna) is widely used in the traditional Ayurvedic system of medicine in cardiac and chronic renal diseases. It is one of the most popular and beneficial medicinal plants for the treatment of cardiovascular diseases. This work provides the latest updates on its traditional use and pharmacological importance, and outlines strategies for future research to develop its clinical potential. The bark of the tree has been widely used in Indian traditional medicine since ancient times, and has been reported to contain several bioactive compounds. Many experimental studies have reported its therapeutic potential in cardiovascular diseases in humans. Many clinical data have reported its efficacy in patients with ischemic heart disease, hypertension, and heart failure. Now is the time for pharmacological researchers to evaluate the chemical constituents of this plant in order to assess its future use in healthcare.
\end{abstract}

Keywords: cardiovascular disorders, medicinal plants, Terminalia arjuna

An herb is a plant or part of a plant used for its therapeutic properties. Herbal medicines are also considered good dietary supplements. People use herbal medicinal foods to try to maintain or improve their health. Nowadays, people have started to use alternative medicines and natural products for basic treatment. Terminalia arjuna is one of the herbal products that has been used as a traditional remedy for many years for the treatment of cardiovascular diseases. Arjuna (Terminalia arjuna Wight \& Arn) is an important medicinal tree known throughout the Indian subcontinent since the Vedic period (1700-550 BCE) (Jain et al. 2009). The bark, leaves, and fruits - almost all the parts of T. arjuna-have been used in Indian traditional medicine for the treatment of many systemic ailments, notably in heart diseases (Warrier et al. 1996; Nema and Garg, 2011). Arjuna bark extract has also been reported to contain numerous functional constituents, e.g., tannins, triterpenoids, saponins (arjunic acid, arjunolic acid, arjungenin, and arjunglycosides), flavonoids (arjunolone, arjunon, and luteolin), gallic acid, ellagic acid, oligomeric Proanthocyanidins (OPCs), Calcium, Magnesium, Zinc, and Copper
(Miller, 1998; Muthu et al. 2006). It has been observed that Arjuna bark decreases the levels of serum triglycerides and cholesterol, recovers the level of high-density lipoprotein (HDL), acts as an anti-ischemic agent, relieves myocardial necrosis, modulates platelet aggregation and acts as an effective antioxidant (Sumitra et al. 2001).

\section{MATERIALS AND METHODS}

Terminalia arjuna, Arjuna for short, is a tree bark that is used to treat cardiovascular problems. The study area was Mehsana and Gandhinagar districts, which are located in Gujarat, India. This location is surrounded by both rural and urban areas. People in this region are aware of options to treat medical emergencies. However, many of them are dependent on herbal products due to poverty and illiteracy. Many villages also have herbal practitioners who advise on traditional methods

How to cite this article: Dhruti, S. (2020). The Use of Terminalia arjuna as a Tonic: A Review. International Journal of Bioresource Science, 7(2): 59-61.

Source of Support: None; Conflict of Interest: None 
of healthcare. Regular field studies, a community study near Gandhinagar district in Gujarat, visits to more than 65 villages, regular conversations with herbal medicine practitioners, and systematic literature searches were carried out during the study preparation. All the available information on Terminalia arjuna was gathered for a detailed study. Terminalia arjuna is widely used in cardiovascular disorders in the form of a powder or syrup. More information about its use was gathered from an urban area where more than 100 families were referred. The collected data were verified through an electronic search using Pubmed, SciFinder, Google Scholar, JCCC@INSTIRC and Web of Science. Some of the literature on Ayurveda was also referred to.

\section{RESULTS AND DISCUSSION}

Arjun is a large deciduous evergreen tree with strong, long roots. Its Sanskrit synonyms are Arjuna, Viravriksha, Kukubha, Partha. Terminalia arjuna (Roxb.) wt. and am: a review. The botanical name of Arjuna is Terminalia arjuna and it belongs to the Combretaceae family. It is commonly known as Arjuna. It has conical leaves, yellow flowers, and smooth gray bark. It has fibrous woody fruit with hard wings and curved veins. The leaves are dull green above and pale brown beneath. The flowers of Arjuna appear between March and July and the fruit between September and November. Terminalia chebula and Terminalia billerica are other species like Arjuna found in India. The bark is used as a cardiac tonic (Kapoor et al. 1994; Mandal et al. 2010).

\section{Chemical constituents}

Arjuna has good antioxidant properties that help to slow down age-related changes in the human body. The important phytochemicals found in this plant are arjunic acid, tannins, arjunones, arjunetin, arjun glycosides 1 and 2, arjunic acid, betullinic acid, and ellagic acid. Research shows that Arjuna bark powder contains arjunone; arjunolone; leteilin; gallic acid; ellagic acid; phytosterols; minerals like calcium, magnesium, zinc, copper and triterpine glycosides like Arjunetosides I, Arjunetosides II, Arjunetosides III, Arjunetosides IV, Arjunine, and Arjunetein (Kolodziej and Kiderlen, 2005).

\section{Mode of application}

The dry bark, leaves, and roots of Arjuna are converted to a powder form that is prepared domestically or available from the market. A twice-daily dose has soothing effects on the heart. It is considered the safest mode of application, in combination with allopathic medicine. Some researchers have also come to the conclusion that Arjuna has a high phytochemical content that accounts for its use as a cardiotonic that can boost heart function and heal cardiac injury. Fresh leaf juice is used for the treatment of earache and bark powder for treating heart ailments by the Malabar tribe of Kerala (Muthu et al. 2006; Mandal et al. 2010). Arjuna is a well-known heart tonic and cardioprotective herb. It strengthens the heart muscles and treats cardiac debility. It also increases the coronary artery flow and protects the heart muscles from ischemic damage.

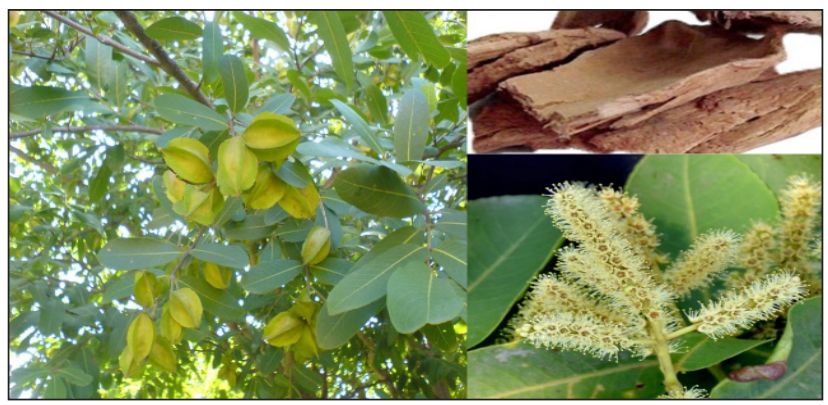

Fig. 1: Terminalia arjuna, Tree bark, Arjuna Flowers

The antioxidant properties allow the use of Arjuna to prevent against some skin-related problems and to treat some diseases like piles, acne, fungal infection, and burns. Local people mix Arjuna powder with milk and honey to make a paste for application (Shahriar et al. 2012).

Fractures: A paste of Arjuna bark, turmeric, and water is applied over fractures to help promote early healing (Prusti and Behera, 2007).

Teeth cleaning: Arjuna twigs are used for teethcleaning. It is said that chewing Arjuna sticks delivers natural compounds directly into the body, which is recommended by local medical professionals (Jain et al. 2009).

High blood pressure: The medicinal herb has diuretic properties, reduces the chances of clot formation, and lowers blood lipids, thereby helping to treat high blood pressure. Tribes living in Sundergarh district, Odisha use dried bark powder along with rice-washed water to treat blood in the urine, and tribes living in Malkangiri district chew 
the fresh bark and swallow the juice as an antacid (Yesodharan and Sujana, 2007).

Cancer treatment: Arjuna herbal medicine contains a substance called casuarinin that seems to prevent breast cancer. T. arjuna is found to downregulate anaerobic metabolism by inhibiting the activity of lactate dehydrogenase in lymphoma-bearing mice; it is elevated in untreated cancerous mice. The results indicate the antioxidant action of an aqueous extract of T. arjuna, which may play a role in the anticarcinogenic activity by reducing oxidative stress and inhibiting anaerobic metabolism (Verma and Vinayak, 2009).

\section{CONCLUSION}

Arjuna is a popular Ayurvedic medicine native to India and south Asia. There is evidence that Terminalia arjuna is efficacious in various cardiovascular disorders. However, more objective, systematic, well-designed research is needed before clinical recommendations can be made regarding Arjuna. Multidisciplinary research is needed to aid the phytochemical study of this plant. Pharmacological research will help with establishing Terminalia arjuna as a potential remedy for cardiovascular diseases and other human health problems. Many Ayurveda practitioners recommend Arjuna tonic and bark powder for cardiac care; it is produced by various pharmaceutical companies and available at most chemists in the Gandhinagar district. Even today, $50-60 \%$ of people in Gandhinagar district and in nearby area uses of Arjuna as a cardiac tonic, whether specifically advised by a doctor to do so or not. Arjuna is one of the plants that has been used in traditional medicine for generations. In both rural and urban areas, people are applying their knowledge about Arjuna to their day-to-day lives.

\section{REFERENCES}

1. Chopra, R.N., Chopra, I.C., Handa, K.L. and Kapur, L.D. 1958. Chopra's Indigenous Drugs of India. $1^{\text {st }}$ ed. Calcutta, India: UN Dhur and Sons; Terminalia arjuna W and A (Combretaceae) pp. 421-4.

2. Dwivedi, S. 2007. Terminalia arjuna Wight and Arn. - A useful drug for cardiovascular disorders. J. Ethnopharmacol., 1: 114-29.
3. Jain, S., Yadav, P.P., Gill, V., Vasudeva, N. and Singla, N. 2009. Terminalia arjuna a sacred medicinal plant: Phytochemical and pharmacological profile. Phytochem. Rev., 8: 491-502.

4. Kapoor D., Vijayvergiya R., Dhawan V. 2014. Terminalia arjuna in coronary artery disease: ethnopharmacology, preclinical, clinical \& safety evaluation. J. Ethnopharmacol., 155: 1029-1045.

5. Kolodziej, H., Kiderlen A.F. 2005. Anti-leishmanial activity and immunemodulatory effects of tannins and related compounds on Leishmania parasitised RAW264.7 cells. Phytochemistry, 66: 2056-2071.

6. Mandal, A., Das, K. and Nandi, D.K. 2010. In vitro bioactivity study of bark extract of Terminalia arjuna on probiotics, commercially available probiotic formulation. Int. J. Phytopharmacol., 1(2): 109-113.

7. Miller, A.L. 1998. Botanical influences on Cardiovascular Diseases. Alt. Med. Rev., 30: 422-431.

8. Muthu, C., Ayyanar, M., Raja, N. and Ignacimuthu, S. 2006. Medicinal plants used by traditional healers in Kancheepuram District of Tamil Nadu, India. J. Ethnobilol. Ethnomed., 2: 43.

9. Prusti, A.B. and Behera, K.K. 2007. Ethno-Medico Botanical Study of Sundargarh district, Orissa, India. Ethnobot. Leafl., 11: 148-63.

10. Shahriar, M., Akhter, S., Hossain, M.I., Haque, M.A. and Bhuiyan, M.A. 2012 Evaluation of in vitro antioxidant activity of bark extracts of Terminalia arjuna. J. Med. Plant Res., 39: 5286-5298.

11. Sharma, S., Sharma, D. and Agarwal, N. 2012. Diminishing effect of Arjuna tree (Terminalia arjuna) bark on the lipid and oxidative stress status of high fat high cholesterol fed rats and development of certain dietary recipes containing the tree bark for human consumption. Res. Pharm., 2: 22-30.

12. Sumitra, M., Manikandan, P., Kumar, D.A., Arutselvan, N., Balakrishna, K., Manohar, B.M. and Puvanakrishnan, R. 2001. Experimental myocardial necrosis in rats: role of arjunolic acid on platelet aggregation, coagulation and antioxidant status. Mol. Cellular Biochem., 224: 135-142.

13. Verma, N. and Vinayak, M. 2009. Effect of Terminalia arjuna on antioxidant defense system in cancer. Mol. Biol. Rep., 36: 159.

14. Warrier, P.K., Nambiar, V.P.K. and Ramankutty, C. 1996. Terminalia arjuna. In: Warrier et al. (eds) In Indian Medicinal Plants - A Compendium of 500 Species. Madras: Orient Longman Ltd., pp. 253-257.

15. Yesodharan, K. and Sujana, K.A. 2007. Ethnomedicinal knowledge among Malamalasar tribe of Parambikulam Wildlife Sanctuary, Kerala. Indian J. Tradit Knowledge, 6: 481-5. 
